\title{
Microscopic Characterization of Heterogeneous Catalysts in 3-D and in-situ/ex- situ
}

Ilke Arslan ${ }^{1}$, Sanchita Dey ${ }^{2}$, John D. Roehling ${ }^{2}$, K. Joost Batenburg ${ }^{3}$, Burtron H. Davis ${ }^{4}$, Bruce C. Gates $^{2}$, Alexander Katz ${ }^{5}$, Daniel E. Perea ${ }^{1}$, and Johannes Lercher ${ }^{1,6}$

${ }^{1}$ Pacific Northwest National Laboratory, Richland, WA 99352, USA

${ }^{2}$ University of California-Davis, Davis, CA 95616, USA

${ }^{3}$ University of Antwerp, Antwerp, Belgium, and Centrum Wiskunde \& Informatica, Amsterdam, The Netherlands

${ }^{4}$ Center for Applied Energy Research, University of Kentucky, Lexington, KY 40511, USA

${ }^{5}$ University of California-Berkeley, Berkeley, CA 94720, USA

${ }^{6}$ TU München, Department of Chemistry, Garching, Germany

The atomic, nanoscale, and three dimensional (3-D) properties of catalysts play an important role in their ultimate activity and selectivity as industrial materials. However, it is also important for these properties to be ascertained as close to reaction conditions as possible. This study brings together advanced methods in the scanning transmission electron microscope and the atom probe with a series of ex-situ and in-situ treatments to understand the fundamental properties of zeolites and FischerTropsch (FT) catalysts.

Zeolites are important due to their wide range of regular pore structures and catalytic site geometries that allows tuning of the catalytic properties. This means that identifying the active site locations and density is important, as well as providing a pore size or surface area large enough for the molecules of interest to enter and react. If a large enough pore size cannot be found, delamination of a layered zeolite may become necessary [1]. I n FT catalysis, it is important to understand the size, morphology, and distribution of the active metal species within its support. We will show this can be accomplished using a combination of 3-D electron tomography and ex-situ gas reactions.

Electron (STEM) tomography was performed on three sets of materials to determine the morphology of the delamination process. The starting material is MCM-22 (P), which has been shown in the literature, and reproduced here, to consist of stacks of many flat layers. Figure 1 (top) shows the 3-D reconstruction, providing a 3-D visualization of the stacks of flat layers. Upon treating the MCM-22 chemically in an aqueous solution at a $\mathrm{pH}$ of 9 [2], a new material is formed, which we call UCB-1 uncalcined. The 2-D and 3-D characteristics of this material are similar to MCM-22 in that the layers of zeolite are still flat and closely packed. However, it is thought that the chemical treatment has acted to break many of the bonds between the zeolitic sheets because when UCB-1 is calcined at 823 $\mathrm{K}$, a very different morphology emerges. It is different from the previous materials in two ways: the first is that the layers are curved and peeled open, exposing much more surface area than the previous materials. The second is that the distance between clusters is also larger, which again provides higher surface area and bigger spaces for bulky molecules to enter and react $[3,4]$. 
[1] A. Corma, et al., Nature, 396, (1998) 353.

[2] I. Ogino, et. al. J. Am. Chem. Soc., 133, (2011) 3288.

[3] I. Arslan and E. A. Stach, Nature Materials, 11, (2012) 911.

[4] This research was funded in part by the DOE BES DE-SC0005822, the ACS Petroleum Research Fund, and the LDRD and Chemical Imaging Initiative programs at PNNL. The Pacific Northwest National Laboratory is operated by Battelle under contract DE-AC05-76RL01830.
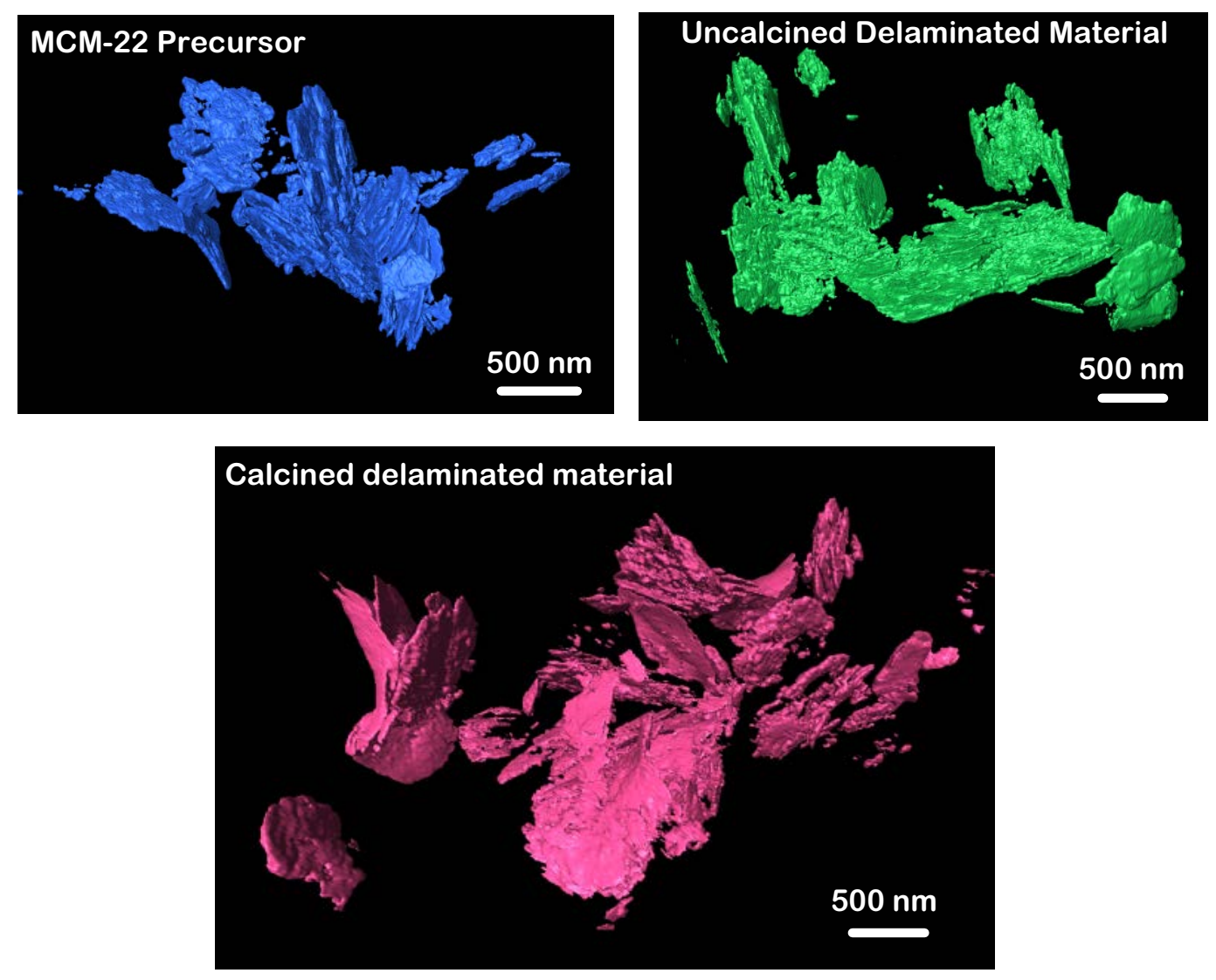

Figure 1. 3-D electron tomographic reconstructions showing the evolution of morphology during the delamination process of a zeolite. 